\title{
Non-Gatherable Triples for Non-Affine Root Systems ${ }^{\star}$
}

\author{
Ivan CHEREDNIK and Keith SCHNEIDER
}

Department of Mathematics, UNC Chapel Hill, North Carolina 27599, USA

E-mail: chered@email.unc.edu,schneidk@email.unc.edu

Received September 03, 2008, in final form November 08, 2008; Published online November 14, 2008

Original article is available at http://www.emis.de/journals/SIGMA/2008/079/

\begin{abstract}
This paper contains a complete description of minimal non-gatherable triangle triples in the lambda-sequences for the classical root systems, $F_{4}$ and $E_{6}$. Such sequences are associated with reduced decompositions (words) in affine and non-affine Weyl groups. The existence of the non-gatherable triples is a combinatorial obstacle for using the technique of intertwiners for an explicit description of the irreducible representations of the (double) affine Hecke algebras, complementary to their algebraic-geometric theory.
\end{abstract}

Key words: root systems; Weyl groups; reduced decompositions

2000 Mathematics Subject Classification: 20H15; 20F55

\section{Introduction}

This paper is a continuation of the part of [2] devoted to non-gatherable triangle triples, NGT, in $\lambda$-sequences. The latter are the sequences of positive roots associated with reduced decompositions (words) in affine and non-affine Weyl groups. We demonstrate that minimal NGT can be completely described in the non-affine case; the answer appears especially simple for the classical root systems and for $F_{4}, E_{6}$ (there are no NGT for $A_{n}, B_{2}, C_{2}, G_{2}$ ). As for $F_{4}, E_{6,7,8}$, we reduced the problem to certain verifications performed by computer; it will be discussed in further works, as well as affine generalizations.

The existence of NGT is a combinatorial obstacle for using the technique of intertwiners (see, e.g. [2]) for an explicit description of the irreducible representations of the affine (and double affine) Hecke algebras, complementary to the geometric theory of [7]. However, NGT are interesting in their own right. Gathering together the triangle triples using the Coxeter transformations seems an important question in the theory of reduced decompositions of Weyl groups, which is far from being simple. More generally, assuming that $\lambda(w)$ contains all positive roots of a root subsystem, can they be gathered using the Coxeter transformations?

Let $R \in \mathbb{R}^{n}$ be a reduced irreducible root system or its affine extension, $W$ the corresponding Weyl group. Then the $\lambda$-set is defined as $\lambda(w)=R_{+} \cap w^{-1}\left(-R_{+}\right)$for $w \in W$, where $R_{+}$is the set of positive roots in $R$. It is well-known that $w$ is uniquely determined by $\lambda(w)$; many properties of $w$ and its reduced decompositions can be interpreted in terms of this set. The $\lambda$ sequence is the $\lambda$-set with the ordering of roots naturally induced by the corresponding reduced decomposition.

The intrinsic description of such sets and sequences is given in terms of the triangle triples $\{\beta, \gamma=\alpha+\beta, \alpha\}$. For instance, $\alpha, \beta \in \lambda(w) \Rightarrow \alpha+\beta \in \lambda(w)$ and the latter root must appear between $\alpha$ and $\beta$ if this set is treated as a sequence. This property is necessary but not sufficient; here and below see [2] for a comprehensive discussion.

We need to know when a set of positive roots of a rank two subsystem inside a given sequence $\lambda(w)$ can be gathered (made consecutive) using the Coxeter transformations in $\lambda(w)$. It

\footnotetext{
${ }^{\star}$ This paper is a contribution to the Special Issue on Kac-Moody Algebras and Applications. The full collection is available at http://www.emis.de/journals/SIGMA/Kac-Moody_algebras.html
} 
is natural to allow the transformations only within the minimal segment containing these roots. This problem can be readily reduced to considering the triangle triples $\{\beta, \gamma=\alpha+\beta, \alpha\}$ provided some special conditions on the lengths. The answer is always affirmative only for the root systems $A_{n}, B_{2}, C_{2}, G_{2}$ (and their affine counterparts) or in the case when $|\alpha| \neq|\beta|$; otherwise NGT always exist.

For the root system $A_{n}$, gathering the triples is simple. It readily results from the planar interpretation of the reduced decompositions and the corresponding $\lambda$-sequences in terms of $(n+1)$ lines in the two-dimensional plane. This interpretation is essentially equivalent to the classical geometric approach to the reduced decompositions of $w \in W$ in terms of the lines (or pseudo-lines) that go from the main Weyl chamber to the chamber corresponding to $w$; see [1].

The $A_{n}$-planar interpretation was extended in [3] to other classical root systems and $G_{2}$, and then to their affine extensions in [4]. It is given in terms of $n$ lines in $\mathbb{R}^{2}$ with reflections in one or two "mirrors" for $B_{n}, C_{n}, D_{n}$ (two mirrors are needed in the affine case) or in terms of $(n+1)$ lines on the two-dimensional cylinder for the affine $A_{n}$.

We use the planar interpretation for the non-affine systems $B, C, D$ to find all minimal non-gatherable triples, minimal $N G T$, in these three cases. No such interpretation is known for $F_{4}, E_{6,7,8}$, but we managed to calculate all minimal NGT in these cases as well. The affine root systems will be considered in the next paper.

Generally, the admissibility condition from [2] is necessary and sufficient for the triple to be gatherable, which is formulated in terms of subsystems of $R$ of types $B_{3}, C_{3}$ or $D_{4}$. We (re)establish this theorem in the non-affine case in this paper and make the proof very constructive. The proof presented in [2] was entirely algebraic, not quite complete for the system $F_{4}$ and sketchy in the $D, E$-cases.

It is important to note that the existence of NGT and other facts of similar nature are in sharp contrast with the case of $A$. Generally, the theory of root systems is uniform at level of generators and relations of the corresponding Weyl or braid group; however the root systems behave differently when the "relations for Coxeter relations" are considered, i.e., at level of the second fundamental group.

Presumably, the phenomenon of NGT is one of the major combinatorial obstacles for creating a universal theory of AHA-DAHA "highest vectors" generalizing Zelevinsky's segments in the $A$-case and based on the intertwining operators. This technique was fully developed only for affine and double affine Hecke algebras of type $A_{n}$ and in some cases of small ranks.

The classification and explicit description of semisimple representations of AHA and DAHA is a natural application of this technique. The fact that all triples are gatherable in the case of $A_{n}$ was important in [5] and in further papers on the quantum fusion procedure; in type A, AHA and DAHA are dual to quantum groups and quantum toroidal algebras, generalizing affine Kac-Moody algebras. Extending the technique of intertwiners to other root systems requires a thorough analysis of NGT.

\section{Weyl groups}

Let $R=\{\alpha\} \subset \mathbb{R}^{n}$ be a root system of type $A, B, \ldots, F, G$ with respect to a Euclidean form $\left(z, z^{\prime}\right)$ on $\mathbb{R}^{n} \ni z, z^{\prime}, W$ the Weyl group generated by the reflections $s_{\alpha}, R_{+}$the set of positive roots corresponding to fixed simple roots $\alpha_{1}, \ldots, \alpha_{n}, \Gamma$ the Dynkin diagram with $\left\{\alpha_{i}, 1 \leq i \leq n\right\}$ as the vertices. We will sometimes use the dual roots (coroots) and the dual root system:

$$
R^{\vee}=\left\{\alpha^{\vee}=2 \alpha /(\alpha, \alpha)\right\} .
$$

Let $\theta \in R^{\vee}$ be the maximal positive root, $\vartheta \in R^{\vee}$ the maximal short root. The latter root is also the maximal positive coroot under the normalization $(\alpha, \alpha)=2$ for short roots. Recall that $1 \geq\left(\theta, \alpha^{\vee}\right) \geq 0$ for $\theta \neq \alpha>0$. Similarly, $1 \geq\left(\vartheta, \alpha^{\vee}\right) \geq 0$ for $\vartheta \neq \alpha>0$. 
Note that the sum of the long roots is always long, the sum of two short roots can be a long root only if they are orthogonal to each other.

The length of the reduced decomposition of $w \in W$ in terms of the simple reflections $s_{i}=s_{\alpha_{i}}$ $(1 \leq i \leq n)$ is denoted by $l(w)$. It can be also defined as the cardinality $|\lambda(w)|$ of the $\lambda$-set of $w$ :

$$
\lambda(w) \stackrel{\text { def }}{=} R_{+} \cap w^{-1}\left(R_{-}\right)=\left\{\alpha \in R_{+}, w(\alpha) \in R_{-}\right\}, \quad w \in W .
$$

The coincidence with the previous definition is based on the equivalence of the length equality

$$
\text { (a) } l_{\nu}(w u)=l_{\nu}(w)+l_{\nu}(u) \quad \text { for } \quad w, u \in W
$$

and the cocycle relation

$$
\text { (b) } \lambda_{\nu}(w u)=\lambda_{\nu}(u) \cup u^{-1}\left(\lambda_{\nu}(w)\right)
$$

which, in its turn, is equivalent to the positivity condition

$$
\text { (c) } u^{-1}\left(\lambda_{\nu}(w)\right) \subset R_{+} \text {. }
$$

Applying (2.3) to the reduced decomposition $w=s_{i_{l}} \cdots s_{i_{2}} s_{i_{1}}$ :

$$
\lambda(w)=\left\{\alpha^{l}=w^{-1} s_{i_{l}}\left(\alpha_{i_{l}}\right), \ldots, \alpha^{3}=s_{i_{1}} s_{i_{2}}\left(\alpha_{i_{3}}\right), \alpha^{2}=s_{i_{1}}\left(\alpha_{i_{2}}\right), \alpha^{1}=\alpha_{i_{1}}\right\} .
$$

This relation demonstrates directly that the cardinality $l$ of the set $\lambda(w)$ equals $l(w)$. Cf. [6, Section 4.5]. We also note that $\lambda_{\nu}\left(w^{-1}\right)=-w\left(\lambda_{\nu}(w)\right)$.

It is worth mentioning that a counterpart of the $\lambda$-set can be introduced for reduced decomposition $w=s_{i_{l}} \cdots s_{i_{2}} s_{i_{1}}$ in arbitrary Coxeter groups. Following [1, Ch. IV, 1.4, Lemma 2] one can define

$$
\Lambda(w)=\left\{t_{l}=w^{-1} s_{i_{l}}\left(s_{i_{l}}\right), \ldots, t_{3}=s_{i_{1}} s_{i_{2}}\left(s_{i_{3}}\right), t_{2}=s_{i_{1}}\left(s_{i_{2}}\right), t_{1}=s_{i_{1}}\right\},
$$

where the action is by conjugation; $\Lambda(w) \subset W$.

The $t$-elements are pairwise different if and only if the decomposition is reduced (a simple straight calculation; see [1]); then this set does not depend on the choice of the reduced decomposition. It readily gives a proof of formula (2.5) by induction and establishes the equivalence of (a), (b) and (c).

Using the root system dramatically simplifies theoretical and practical (via computers) analysis of the reduced decompositions and makes the crystallographical case significantly simpler than the case of abstract Coxeter groups. The positivity of roots, the alternative definition of the $\lambda$-sets from (2.1) and, more specifically, property (c) are of obvious importance. These features are (generally) missing in the theory of abstract Coxeter groups, though the $\Lambda$-sets from (2.6) can be of course used for various questions.

The sets $\lambda(w)$ can be treated naturally as a sequence; the roots in (2.5) are ordered naturally depending on the particular choice of a reduced decomposition. We will mainly treat $\lambda(w)$ as sequences in this paper, called $\lambda$-sequences.

Note that relation (2.4) readily gives that an arbitrary simple root $\alpha_{i} \in \lambda(w)$ can be made the first in a certain $\lambda$-sequence. More generally:

$$
\lambda_{\nu}(w)=\left\{\alpha>0, l_{\nu}\left(w s_{\alpha}\right) \leq l_{\nu}(w)\right\} ;
$$

see [1] and [6, Section 4.6, Exchange Condition]. This property is closely related to the formula:

$$
\alpha \in \lambda(w) \Leftrightarrow \lambda\left(s_{\alpha}\right)=\left\{\beta,-s_{\alpha}(\beta) \mid s_{\alpha}(\beta) \in-R_{+}, \beta \in \lambda(w)\right\} .
$$




\section{Coxeter transformations}

We will prepare some tools for studying transformations of the reduced decompositions. The elementary ones are the Coxeter transformations that are substitutions $\left(\cdots s_{i} s_{j} s_{i}\right) \mapsto\left(\cdots s_{j} s_{i} s_{j}\right)$ in reduced decompositions of the elements $w \in W$; the number of $s$-factors is $2,3,4,6$ as $\alpha_{i}$ and $\alpha_{j}$ are connected by $m_{i j}=0,1,2,3$ laces in the affine or non-affine Dynkin diagram. They induce reversing the order in the corresponding segments (with 2, 3, 4, 6 roots) inside the sequence $\lambda(w)$. The corresponding roots form a set identified with the set of positive roots of type $A_{1} \times A_{1}, A_{2}, B_{2}, G_{2}$ respectively.

The theorem below is essentially from [2]; it has application to the decomposition of the polynomial representation of DAHA, the classification of semisimple representations of AHA, DAHA and to similar questions. We think that it clarifies why dealing with the intertwining operators for arbitrary root systems is significantly more difficult than in the $A_{n}$-theory (where much is known).

Given a reduced decomposition of $w \in W$, let us assume that $\alpha+\beta=\gamma$ for the roots $\ldots, \beta, \ldots, \gamma, \ldots, \alpha \ldots$ in $\lambda(w)$ ( $\alpha$ appears the first), where only the following combinations of their lengths are allowed in the $B, C, F$ cases

$$
\operatorname{lng}+\operatorname{lng}=\operatorname{lng}\left(B, F_{4}\right) \quad \text { or } \quad \operatorname{sht}+\operatorname{sht}=\operatorname{sht}\left(C, F_{4}\right)
$$

Since we will use the Coxeter transformations only inside the segment $[\beta, \alpha] \subset \lambda(w)$ between $\beta$ and $\alpha$, it suffices to assume that $\alpha$ is a simple root. Also, let us exclude $A_{n}, B_{2}, C_{2}, G_{2}$ from the consideration (in these cases all triangle triples, if any, are gatherable).

Theorem 1. (i) For the root systems of type $B_{n}, C_{n}, F_{4}$, the roots $\beta, \gamma, \alpha$ are non-gatherable (cannot be made consecutive using the Coxeter transformations) inside the segment $[\beta, \alpha] \subset \lambda(w)$ if and only if a root subsystem $R^{3} \subset R$ of type $B_{3}$ or $C_{3}(m=1,2)$ exists such that

$$
\beta=\epsilon_{1}+\epsilon_{3}, \alpha=\epsilon_{2}-\epsilon_{3}, \epsilon_{1}-\epsilon_{2} \notin[\beta, \alpha] \not \ngtr m \epsilon_{3},
$$

where the roots $\epsilon_{1}, \epsilon_{2}, \epsilon_{3} \in R^{3}$ are from the $B_{3}, C_{3}$ tables of [1]. Equivalently, the sequence $[\beta, \alpha] \cap R_{+}^{3}$ (with the natural ordering) must be

$$
\left\{\epsilon_{1}+\epsilon_{3}, m \epsilon_{1}, \epsilon_{2}+\epsilon_{3}, \gamma=\epsilon_{1}+\epsilon_{2}, \epsilon_{1}-\epsilon_{3}, m \epsilon_{2}, \epsilon_{2}-\epsilon_{3}\right\}
$$

up to Coxeter transformations in $R^{3}$ and changing the order of all roots in (3.3) to the opposite. This sequence is $\lambda^{3}\left(s_{\gamma}\right)$ in $R_{+}^{3}$ for the maximal root $\gamma=\theta^{3}$ for $B_{3}$ and for the maximal short root $\gamma=\vartheta^{3}$ for $C_{3}$.

(ii) For the root system $R$ of type $D_{n \geq 4}$ or for $E_{6,7,8},\{\beta, \gamma, \alpha\}$ is a non-gatherable triple if and only if a root subsystem $R^{4} \subset R$ of type $D_{4}$ can be found such that

$$
\begin{aligned}
& \beta=\epsilon_{1}+\epsilon_{3}, \quad \gamma=\epsilon_{1}+\epsilon_{2}, \quad \alpha=\epsilon_{2}-\epsilon_{3}, \\
& \left\{\epsilon_{1}-\epsilon_{2}, \epsilon_{3}-\epsilon_{4}, \epsilon_{3}+\epsilon_{4}\right\} \cap[\beta, \alpha]=\varnothing,
\end{aligned}
$$

where $\epsilon_{1}, \epsilon_{2}, \epsilon_{3}, \epsilon_{4}$ are from the $D_{4}$-table of [1]. Equivalently, the sequence $[\beta, \alpha] \cap R_{+}^{4}$ must be

$$
\begin{array}{r}
\left\{\beta=\epsilon_{1}+\epsilon_{3}, \epsilon_{1}-\epsilon_{4}, \epsilon_{1}+\epsilon_{4}, \epsilon_{1}-\epsilon_{3}, \gamma=\epsilon_{1}+\epsilon_{2}\right. \\
\left.\epsilon_{2}+\epsilon_{3}, \epsilon_{2}+\epsilon_{4}, \epsilon_{2}-\epsilon_{4}, \alpha=\epsilon_{2}-\epsilon_{3}\right\}
\end{array}
$$

up to Coxeter transformations in $R^{4}$. Equivalently, $[\beta, \alpha] \cap R_{+}^{4}$ is the $\lambda$-set of $s_{\theta^{4}}$ in $R_{+}^{4}$ for the maximal root $\theta^{4}$. 
We will (re)prove this theorem (later) by listing all minimal non-gatherable triples. Our approach is significantly more explicit than that from [2], although Theorem 1 does not require the minimality condition and therefore is of more general nature. The affine root systems will be considered elsewhere.

To begin with, the following are the lists of the non-affine roots $\gamma>0$ such that the endpoints $\beta=\gamma-\alpha_{j}, \alpha=\alpha_{j}$ of $\lambda\left(s_{\gamma}\right)$ are non-movable under the Coxeter transformations within $\lambda\left(s_{\alpha}\right)$ and $\{\beta, \gamma, \alpha\}$ form an $A_{2}$-triple in the cases of $F_{4}, B, C ; A_{2}$-triples are those subject to $\left|\alpha_{j}\right|=$ $|\gamma|=|\beta|$. See [2] and also (2.8). The maximal long root $\theta$ (for $B_{n \geq 3}, F_{4}$ and in the simply-laced case) and maximal short root $\vartheta$ (for $C_{n \geq 3}, F_{4}$ ) are examples of such $\gamma$ (but there are many more).

The bar will show the position of the corresponding $\alpha_{j}$ in the Dynkin diagram. We will omit the cases of $E_{7,8}$; there are 7 such $\gamma$ for $E_{7}$ and 22 for $E_{8}$.

The case of $\boldsymbol{E}_{\mathbf{6}}$. The roots $\gamma \in R_{+}$such that $\lambda\left(s_{\gamma}\right)$ has non-movable endpoints are:

$\begin{array}{ccccc}01 \overline{2} 10, & 1 \overline{2} 210, & 012 \overline{2} 1, & 12 \overline{3} 21, & 12321 . \\ 1 & 1 & 1 & 1 & \overline{2}\end{array}$

The corresponding triple $\left\{\beta=\gamma-\alpha_{j}, \gamma, \alpha_{j}\right\}$ is a minimal non-gatherable triple inside $\lambda\left(s_{\gamma}\right)$.

The case of $\boldsymbol{F}_{\mathbf{4}}$. The roots $\gamma \in R_{+}$with non-movable endpoints of $\lambda\left(s_{\gamma}\right)$ and subject to $\left|\alpha_{j}\right|=|\gamma|$ are:

$01 \overline{2} 1, \quad 1 \overline{2} 20, \quad 12 \overline{3} 1, \quad 123 \overline{2}, \quad 1 \overline{3} 42, \quad \overline{2} 342$.

The case of $\boldsymbol{B}, \boldsymbol{C}, \boldsymbol{D}_{\boldsymbol{n}}$. Given $\alpha_{j}=\epsilon_{j}-\epsilon_{j+1}$, the corresponding root $\gamma$ (it is unique) equals $\epsilon_{j-1}+\epsilon_{j}$ for $j=2, \ldots, n-1$ provided that $n \geq 3$ and $j<n-1 \geq 3$ for $D_{n}$. The notation is from [1].

\section{Minimal NGT}

We are now in a position to formulate the main result of the paper, that is a description of all minimal non-gatherable triples, NGT, for the non-affine root systems. It provides a direct justification of Theorem 1 in the non-affine case. We will omit the lists in the cases $E_{7,8}$ in this paper (there are no NGT for $A_{n}, B_{2}, C_{2}, G_{2}$ ).

We say that $w \in W$ represents a minimal $N G T$, if $\{\beta, \gamma=\alpha+\beta, \alpha\} \subset \lambda(w), \alpha, \beta$ are correspondingly the beginning and the end of the sequence $\lambda(w)$ and these roots are non-movable inside $\lambda(w)$ using the Coxeter transformations. Recall that the condition from (3.1) is imposed.

Theorem 2. The lists of elements $w \in W$ representing minimal $N G T$ are as follows.

(i) For $B_{n}, C_{n}, D_{n}$ an arbitrary $\gamma=\epsilon_{i}+\epsilon_{j}, i<j$, subject to $j<n$ for $B, C$ and $j<n-1$ for $D$ can be taken; the corresponding simple $\alpha$ will be $\epsilon_{j-1}-\epsilon_{j}$ in the notation from [1]. The element $w$ is the product of reflections corresponding to the "telescoping" sequence $\epsilon_{i}+\epsilon_{j}, \epsilon_{i+1}+\epsilon_{j-1}, \ldots$ ending with $\epsilon_{k}+\epsilon_{k+1}$ unless the last root of this sequence is in the form $\epsilon_{k-1}+\epsilon_{k+1}$. In the latter case, the roots $\epsilon_{k}$ or $2 \epsilon_{k}$ must be added to this sequence for $B_{n}$ or $C_{n}$, and the pair of roots $\epsilon_{k}+\epsilon_{n}, \epsilon_{k}-\epsilon_{n}$ must be added for $D_{n}$.

Such $w$ is determined uniquely by $\{i, j\}$ and is a product of reflections for pairwise orthogonal roots; in particular, $w^{2}=\mathrm{id}$. One of these roots must be $\gamma$ (cf. the description of $w=s_{\gamma}$ given above).

(ii) In the case of $F_{4}$, such $w$ are products of pairwise commutative reflection as well, but $w$ is not uniquely determined by the triple. Omitting two $w$ that come from $B_{3}$ and $C_{3}$ naturally embedded into $F_{4}$, the remaining eight are as follows: there are four that are simply reflections with respect to the roots 1231, 1342, 1232, and 2342 from (3.7); the remaining four are each the 
product of reflections of two orthogonal roots, $s_{1342} s_{1110}, s_{0122} s_{1231}, s_{1222} s_{1231}$, and $s_{1342} s_{1121}$. Note that since $F_{4}$ is self dual, the dual of every word on this list is also on this list.

(iii) In the case of $E_{6}$ we will omit 5 elements coming from the two natural $D_{5}$-subsystems of $E_{6} \cdot\left(3+3\right.$ minus the one for $D_{4}$ they have in common; see (i)). Of the remaining ten, two are reflections of the roots $\begin{array}{cccc}12321 & 12321\end{array}$ and $\begin{array}{cc}1 \\ 2\end{array}$ from (3.6).

Two more can be written as the composition of three pairwise orthogonal reflections of roots:

$$
s\left(\begin{array}{c}
12321 \\
1
\end{array}\right) s\left(\begin{array}{c}
01100 \\
1
\end{array}\right) s\left(\begin{array}{c}
00110 \\
1
\end{array}\right), \quad s\left(\begin{array}{c}
01221 \\
1
\end{array}\right) s\left(\begin{array}{c}
12210 \\
1
\end{array}\right) s\left(\begin{array}{c}
11211 \\
1
\end{array}\right) .
$$

The final six can not be written as products of orthogonal reflections. Written as products of reflections of simple roots they are as follows:

$$
\begin{array}{ll}
21324354632143254363243, & 32143263214325436321432, \\
32435463214325436324354, & 43215432632143254363243, \\
2132435463214325436324354, & 4321543263214325436321432,
\end{array}
$$

where we abbreviate $s_{\alpha_{i}}$ as $i$ for $1 \leq i \leq 6$; for instance, the first word is $s_{2} s_{1} \cdots s_{4} s_{3}$.

Proof. We will use the planar interpretation of the reduced decompositions from [3] for $B, C, D$. An arbitrary element $w \in W$ can be represented by a configuration of $n$ lines in the plane with a possible reflection in the $x$-axis. The initial numeration of the lines is from the top to the bottom (the right column of the line numbers in the figures below). The intersections and reflections are supposed to have pairwise different $x$-projections; simultaneous multiple events (like double intersections) are not allowed.

Given an intersection, we plot the vertical line through it and count the lines (locally) from the top; the intersection of the (neighboring) lines $k, k+1$ is interpreted as $s_{k}$. The angle between these lines gives the corresponding root in $\lambda(w)$. Namely, it is $\epsilon_{i} \pm \epsilon_{j}$ for the initial (global) numbers of the lines and their initial angles $\epsilon_{i}, \epsilon_{j}$ with the $x$-axis; the sign depends on the number of reflections before the intersection. See Fig. 1, where we put $i$ instead of $\epsilon_{i}$ in the angles/roots and instead of $s_{i}$ in the decomposition.

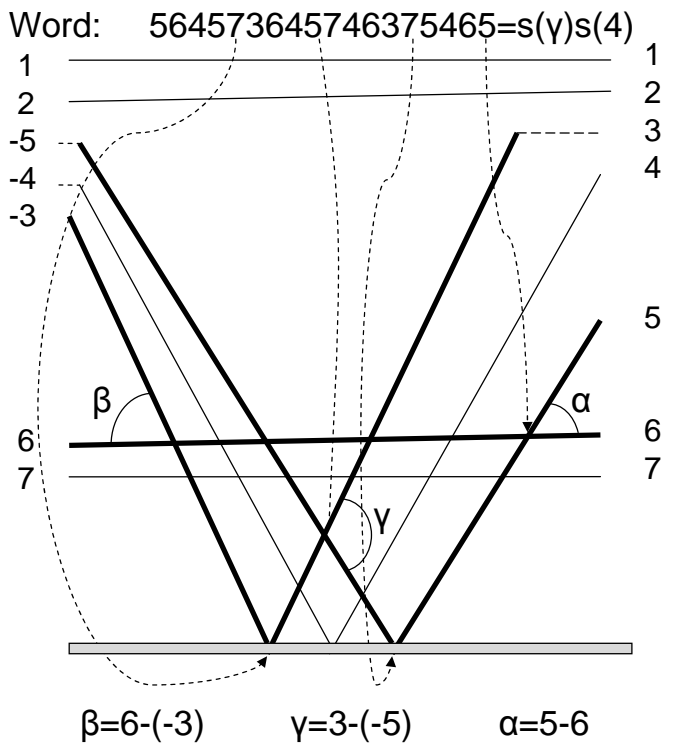

Figure 1. Typical minimal NGT for $B_{7}$. 


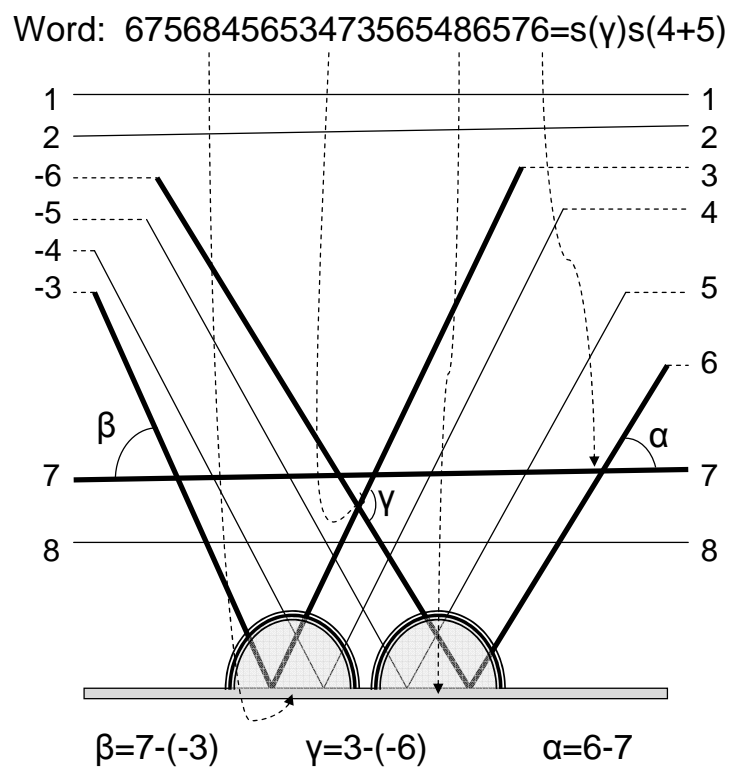

Figure 2. Typical minimal NGT for $D_{8}$.

The angle is taken $\epsilon_{i}$ or $2 \epsilon_{i}$ for the reflection in the $x$-axis in the cases of $B$ or $C$; the corresponding event is interpreted as $s_{n}$ in the Weyl group.

Treating the reflections is a bit more involved in the $D$-case. The combination of the reflection, then the $\{n-1, n\}$-intersection (the numbers of lines are local), and then the reflection again is interpreted as $s_{n}$ for $D_{n}$. The corresponding root from $\lambda(w)$ is the middle angle in this event, which will be called $V \times V$-shape. These events are encircled in Fig. 2; they look like W. Their angles are $5+6\left(\epsilon_{5}+\epsilon_{6}\right.$, to be exact) and $3+4$ correspondingly (from right to left).

This construction is sufficient for constructing reduced decompositions for arbitrary configurations with the even number of reflections in the $x$-axis. Indeed, proper moving the lines upward makes any given configuration a sequence of the simple crosses of lines and the $V \times V$-shapes. However, the geometric interpretation of the Coxeter relation $s_{n-2} s_{n} s_{n-2}=s_{n} s_{n-2} s_{n}$ requires an extra $V+V$-operation, that is breaking a given line twice and adding two reflections, as shown in Figs. 3 and 4 , followed by creating the $V \times V$-shapes. Symbolically, it can be represented by $\mathrm{W}$ (line 4 in Fig. 3). More formally,

1) given a line, this transformation must not increase the total number of its intersections with the other lines;

2) two reflections must exist in a given configuration neighboring to the (new) reflections from $V+V$;

3 ) the pairs of neighboring reflections from (2) have to be arranged into two $V \times V$-shapes.

Performing one such $V+V$ or multiple operations of this type and moving the lines if necessary, the final configuration can be represented in terms of (simple) intersections and $V \times V$-shapes, provided that the number of initial reflections is even. Fig. 3 gives the simplest minimal NGT represented with and without transforming line 4 . Here one avoids breaking line 4 and adding the $V+V$-shape to this line by moving it up (the second picture). Disregarding line 3 , the figure represents the Coxeter relation $s_{n} s_{n-2} s_{n}=s_{n-2} s_{n} s_{n-2}$.

The claim is that the resulting products of simple reflections (the words in the figures) are always reduced; the angles give the corresponding $\lambda$-sequence. Recall, that the ordering of the $x$-projections is from right to left as well as that of the simple reflections and angles. 


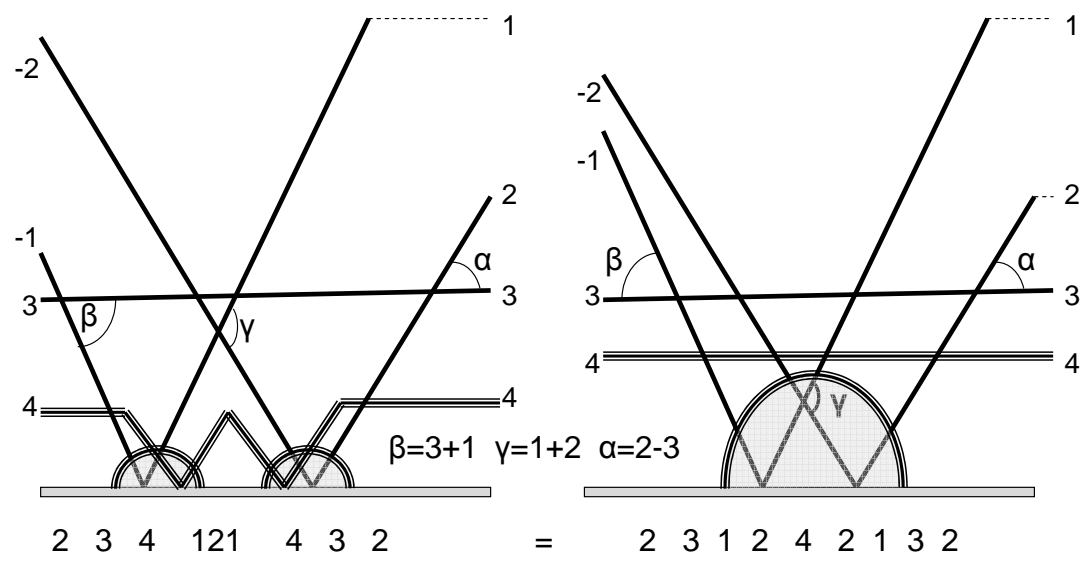

Figure 3. Transforming the line for $D_{4}$.

It is natural to change the global numbers of the lines from $i$ to $-i$ upon the reflections. Then the resulting column of global line numbers (on the left) gives the standard one-line presentation of the corresponding $w$. For the $D$-system, the $V+V$ operation does not change the global numbers at the ends, since we changes the sign two times at the additional reflection points. Note that, technically, we do not change the line number (the "global angle" assigned to this line) at the beginning and at the end of the additional $V+V$-shape; these are "no-events", though, geometrically, the angle of this line is changed at these points.

It is worth mentioning that not all reduced decompositions of $w \in W$ can be obtained using the lines only; generally, one should consider "pseudo-lines", where the assignment of the "global angle" to the line is combinatorial rather than geometric. Fig. 3 is a good demonstration of this phenomenon (the counterexamples exist even for $A_{n}$ with sufficiently big $n$ ).

Using the planar interpretation, the proof of (i) goes as follows.

First of all, any (triangle) triple for $A_{n}$ can be readily made consecutive, corresponding to a "minimal" triangle, using proper parallel translations of the lines. The same argument shows that the root $\gamma$ cannot be $\epsilon_{i}-\epsilon_{j}$ for $B, C, D$ in the notation from [1]. Otherwise, we can make the corresponding triangle "minimal" as for $A_{n}$.

We will consider the $B, C$-case only; the root system $D$ is completely analogous. Given $\gamma=\epsilon_{i}+\epsilon_{j}$, there are three groups of the (initial) lines:

(a) beyond $i$ (lines 1, 2 in Fig. 1),

(b) between $i$ and $j$ (lines 3, 4, 5 there) and

(c) the lines strictly below $j$ (namely, lines 6, 7).

The lines from group (a) do not intersect each other. Otherwise, we can move the intersection point to the right using the parallel translations (as in the $A$-case) and make the corresponding root the first in $\lambda(w)$, which is impossible since $\alpha$ is the only such root. Also the lines from (a) cannot intersect the lines from group (b). If such an intersection occurs then we can move it to the right or to the left till the very first or very last position in a reduced decomposition of $w$, which contradicts to the minimality of the NGT under consideration.

Similarly, the lines from group (c) cannot intersect each other. They also do not intersect the lines from group (a). Moreover, the intersections inside the group (b) can occur only due to the reflections (i.e., all pairs of lines in this group must intersect exactly once). If, say, lines 4 and 5 intersect before the reflection point of line 5 or after the reflection point of line 4 (see the $B$-figure), then we can move this intersection to the right or to the left all the way. Finally, the group (c) is always nonempty (i.e., $j<n$ ); otherwise $s_{n}$ can be made the first in a certain reduced decomposition of $w$. 
It implies that the simple root $\alpha$ (from the triple under consideration) can be only $\epsilon_{j}-\epsilon_{j+1}$, i.e., the one corresponding to the intersection of lines $j$ and $j+1$. Indeed, the other possibility for $\alpha$, the simple root $\epsilon_{i}-\epsilon_{i+1}$, would contradict the minimality of NGT. Respectively, $\beta=\epsilon_{i}+\epsilon_{j+1}$.

Summarizing, $w$ can be only of the type shown in Fig. 1. Geometrically, it is obvious that $\{\beta, \gamma, \alpha\}$ there (and in general) is a minimal non-gatherable triple. Indeed, the endpoints are nonmovable using the Coxeter transformations, which correspond to moving (maybe with changing the angles) the lines without altering their initial and final orderings, i.e., the right and left columns of numbers.

The same reasoning gives that $j<n-1$ and that minimal NGT can be only as shown in Fig. 2 in the $D$-case. This concludes (i).

The lists (ii), (iii) are reduced to certain direct computer calculations to be discussed elsewhere (including the complete lists for $E_{7,8}$ ). We note that finding all $w$ representing minimal NGT for $F_{4}, E_{6}$ is a relatively simple problem for products of pairwise commutative reflections (it is not always true for $\left.E_{6,7,8}\right)$. It is a straightforward generalizations of the description of the pure $w=s_{\gamma}$ representing minimal NGT we gave above. One of these reflections must be $s_{\gamma}$ for $\gamma$ from the triple; it simplifies the consideration.

\section{The existence of $R^{3,4}$}

The Theorem 1 guarantees the existence of the subsystems $B_{3}$ or $C_{3}$ and $D_{4}$, ensuring that the corresponding (admissible) triple is non-gatherable. Recall that the intersection of the (positive roots of) these subsystems with $\lambda(w)$ containing such triple, must contain 7 but not 9 (the total) roots for $B_{3}, C_{3}$ and 9 but not 12 (the total) roots in the case of $D_{4}$. We will call such 7-subsets or 9-subsets in $\lambda(w)$ blocks for $N G T$, respectively, $B_{3}$-blocks, $C_{3}$-blocks, $D_{4}$-blocks.

The blocks can be naturally seen geometrically in the cases $B_{n}, C_{n}$ and $D_{n}$. Indeed, if one considers only bold lines in Fig. 1, then it readily leads to the desired $R^{3}$ in the cases $B_{n}, C_{n}$. The intersection of the $\lambda$-sequence with this $R^{3}$ will contain exactly 7 roots (from possible 9 ), i.e., form a block, an obstacle for gathering the corresponding triple.

For finding a root subsystem $R^{4}$ of type $D_{4}$ in Fig. 2, lines $3,6,7,8$ must be taken. Line 8 must be moved up to make it beyond $\gamma$ (but below line 7) or transformed by adding the $V+V$ shape. It is shown in Fig. 4. If there are several lines like 8 "almost parallel" to each other, then either one can be taken to construct a $D_{4}$-block.

The number of the roots (only positive ones matter) in the intersection of such $D_{4}$-subsystem with the $\lambda$-sequence is always exactly 9 (from possible 12 ), i.e., this intersection is a $D_{4}$-block.

We conclude that the $R^{3}$-subsystem and the corresponding block is unique for a given nongatherable triple in types $B_{n}, C_{n}$. In the case of $D_{n}, a D_{4}$-block always exists for $N G T$, but the $R^{4}$-subsystem is generally not unique. It proves Theorem 1 for the classical roots systems and makes explicit the constructions of blocks.

The case of $\boldsymbol{F}_{\mathbf{4}}$. Given a word $w$ containing a minimal NGT where all three roots are long, Theorem 1 states that there exist three roots, $\alpha_{1}, \alpha_{2}, \alpha_{3}$, with the following properties:

1. The roots $\alpha_{1}, \alpha_{2}, \alpha_{3}$ satisfy the properties of the set of simple roots of a $B_{3}$ root system using notation from [1].

2. The NGT in $\lambda(w)$ can be written $\beta=\alpha_{1}+\alpha_{2}+2 \alpha_{3}, \gamma=\alpha_{1}+2 \alpha_{2}+2 \alpha_{3}, \alpha=\alpha_{2}$.

3. The seven roots $\alpha_{2}, \alpha_{1}+\alpha_{2}, \alpha_{2}+\alpha_{3}, \alpha_{1}+\alpha_{2}+\alpha_{3}, \alpha_{2}+2 \alpha_{3}, \alpha_{1}+\alpha_{2}+2 \alpha_{3}$, and $\alpha_{1}+2 \alpha_{2}+2 \alpha_{3}$ form the intersection of $\lambda(w)$ and the subsystem $R^{3} \subset R$ generated by $\alpha_{1}, \alpha_{2}, \alpha_{3}$.

Note that $\alpha_{2}$ is always a simple root of $F_{4}$ but $\alpha_{1}$ and $\alpha_{3}$ need not be. The two roots $\alpha_{1}$ and $\alpha_{3}$ will not be elements of $\lambda(w)$. Together with the seven roots above these are all nine positive roots in the $R^{3}$, i.e., we come to the condition seven but not nine given in (3.3). 


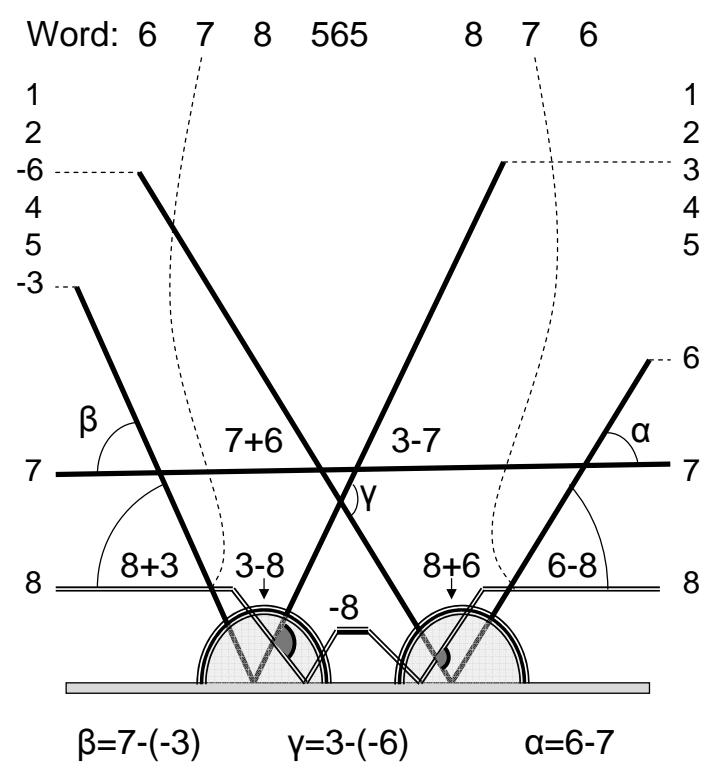

Figure 4. Finding $D_{4}$ in NGT for $D_{8}$.

Table 1. $F_{4}$ Min-NGT's: $B_{3}$-blocks.

\begin{tabular}{|c|c|c|}
\hline$w$ & NGT & $\alpha_{1}, \alpha_{2}, \alpha_{3}$ \\
\hline$s_{1220}$ & $1120,1220,0100$ & $1000,0100,0010$ \\
\hline$s_{1342}$ & $1242,1342,0100$ & $1120,0100,0011$ \\
\hline$s_{2342}$ & $1342,2342,1000$ & $0100,1000,0121$ \\
\hline$s_{1342} s_{1110}$ & $1242,1342,0100$ & $1122,0100,0010$ \\
\hline$s_{1222} s_{1231}$ & $1122,1222,0100$ & $1000,0100,0011$ \\
\hline
\end{tabular}

Following Theorem 2(ii), we will explicitly demonstrate that Theorem 1 holds for minimal NGT and give an appropriate choice of $\alpha_{1}, \alpha_{2}, \alpha_{3}$ (there is often more than one block). Considering only minimal NGT is obviously sufficient to check Theorem 1.

We begin with the following explicit example. Let $w=s_{1342} s_{1110}$; the shortlex form of $w$ is $2132132432132432=s_{2} s_{1} \cdots s_{4} s_{3} s_{2}$. Here the ordering is lexicographical from left to right (but we apply the corresponding reflections from right to left).

This yields:

$$
\begin{aligned}
\lambda(w)=\{ & 1242,1120,1232,2342,1222,1110,1100,1231, \\
& 1221,1342,1220,0121,0120,0111,0110,0100\} .
\end{aligned}
$$

The NGT is $\{1242,1342,0100\}$. If we choose $\alpha_{1}=1122, \alpha_{2}=0100, \alpha_{3}=0010$, then $\left\{\alpha_{1}+\right.$ $\left.\alpha_{2}+2 \alpha_{3}, \alpha_{1}+2 \alpha_{2}+2 \alpha_{3}, \alpha_{2}\right\}=\{1242,1342,0100\}$ (the NGT), and $\lambda(w)$ contains the $B_{3}$-block:

$$
\begin{gathered}
\left\{\alpha_{2}=0100, \alpha_{1}+\alpha_{2}=1222, \alpha_{2}+\alpha_{3}=0110, \alpha_{1}+\alpha_{2}+\alpha_{3}=1232,\right. \\
\left.\alpha_{2}+2 \alpha_{3}=0120, \alpha_{1}+\alpha_{2}+2 \alpha_{3}=1242, \alpha_{1}+2 \alpha_{2}+2 \alpha_{3}=1342\right\} .
\end{gathered}
$$

Finally, $\lambda(w)$ does not contain either $\alpha_{1}=1122$ or $\alpha_{3}=0010$. So the seven but not nine condition is satisfied.

Table 1 shows each of the Min-NGT words in $F_{4}$ where the NGT is made up of long roots and, correspondingly, the block must be of type $B_{3}$. Also included are the NGT and a choice of $\alpha_{1}, \alpha_{2}, \alpha_{3}$ that determine an appropriate $B_{3}$-block. The word used in the example above is also included.

Short NGT for $\boldsymbol{F}_{4}$. Similarly, if the roots from Min-NGT are all short, then Theorem 1 in the case of $F_{4}$ tells us that there exist three roots, $\alpha_{1}, \alpha_{2}, \alpha_{3}$, with the following properties: 
Table 2. $F_{4}$ Min-NGT: $C_{3}$-blocks.

\begin{tabular}{|c|c|c|}
\hline$w$ & NGT & $\alpha_{1}, \alpha_{2}, \alpha_{3}$ \\
\hline$s_{0121}$ & $0111,0121,0010$ & $0001,0010,0100$ \\
\hline$s_{1231}$ & $1221,1231,0010$ & $0111,0010,1100$ \\
\hline$s_{1232}$ & $1231,1232,0001$ & $0010,0001,1220$ \\
\hline$s_{1231} s_{0122}$ & $1221,1231,0010$ & $1111,0010,0100$ \\
\hline$s_{1121} s_{1342}$ & $1111,1121,0010$ & $0001,0010,1100$ \\
\hline
\end{tabular}

Table 3. $E_{6}$ Min-NGT: $D_{4}$-blocks.

\begin{tabular}{|c|c|c|}
\hline$w$ & NGT & $\alpha_{1}, \alpha_{2}, \alpha_{3}, \alpha_{4}$ \\
\hline 324363243 & $011101,012101,001000$ & $010000,001000,000100,000001$ \\
\hline 2132436321432 & $112101,122101,010000$ & $100000,010000,001000,001101$ \\
\hline 4325436324354 & $012111,012211,000100$ & $001000,000100,000010,011001$ \\
\hline 321432632143263 & $111101,112101,001000$ & $000100,001000,000001,110000$ \\
\hline 324354632435463 & $011111,012111,001000$ & $010000,001000,000001,000110$ \\
\hline 3214325436321432543 & $122211,123211,001000$ & $010000,001000,000100,111111$ \\
\hline 632143254363214325436 & $123211,123212,000001$ & $001000,000001,011100,111110$ \\
\hline 21324354632143254363243 & $122211,123211,001000$ & $010000,001000,000100,111111$ \\
\hline 32143254632143254363243 & $122211,123211,001000$ & $010000,001000,000100,111111$ \\
\hline 32143263214325436321432 & $112111,122111,010000$ & $100000,010000,001000,001111$ \\
\hline 32435463214325436324354 & $112111,112211,000100$ & $001000,000100,000010,111001$ \\
\hline 43215432632143254363243 & $122211,123211,001000$ & $010000,001000,000100,111111$ \\
\hline 2132435463214325436324354 & $122111,122211,000100$ & $000010,000100,011000,111001$ \\
\hline 4321543263214325436321432 & $112211,122211,010000$ & $100000,010000,001100,001111$ \\
\hline 32143254363214325436321432543 & $111111,112111,001000$ & $000001,001000,110000,000110$ \\
\hline
\end{tabular}

1. The roots $\alpha_{1}, \alpha_{2}, \alpha_{3}$ satisfy the properties of the set of simple roots of a $C_{3}$ root system using notation from [1].

2. The NGT in $\lambda(w)$ is written $\alpha_{1}+\alpha_{2}+\alpha_{3}, \alpha_{1}+2 \alpha_{2}+\alpha_{3}, \alpha_{2}$.

3. The intersection $\lambda(w) \cap R^{3}$ is formed by $\alpha_{2}, \alpha_{1}+\alpha_{2}, \alpha_{2}+\alpha_{3}, \alpha_{1}+\alpha_{2}+\alpha_{3}, 2 \alpha_{2}+\alpha_{3}$, $\alpha_{1}+2 \alpha_{2}+\alpha_{3}$, and $2 \alpha_{1}+2 \alpha_{2}+\alpha_{3}$ for $R^{3} \subset R$ generated by $\alpha_{1}, \alpha_{2}, \alpha_{3}$.

Here $\alpha_{2}$ is always a simple root of $F_{4}$ but $\alpha_{1}$ and $\alpha_{3}$ need not be. The two roots $\alpha_{1}$ and $\alpha_{3}$ will not be elements of $\lambda(w)$. Together with the seven roots above these are all nine positive roots of $R^{3}$. This condition seven but not nine from (3.3) is satisfied.

Table 2 shows each of the Min-NGT words in $F_{4}$ where the NGT is made up of short roots. Also included are the roots that make up the NGT and a choice of $\alpha_{1}, \alpha_{2}, \alpha_{3}$ that determines an appropriate $C_{3}$ subsystem.

The case of $\boldsymbol{E}_{\mathbf{6}}$. Due to Theorem 1, given a word $w$ containing a Min-NGT, there exist three roots, $\alpha_{1}, \alpha_{2}, \alpha_{3}, \alpha_{4}$, with the following properties:

1. The roots $\alpha_{1}, \alpha_{2}, \alpha_{3}, \alpha_{4}$ satisfy the properties of the set of simple roots of a $D_{4}$ root system using notation from [1].

2. The NGT in $\lambda(w)$ can be written $\beta=\alpha_{1}+\alpha_{2}+\alpha_{3}+\alpha_{4}, \gamma=\alpha_{1}+2 \alpha_{2}+\alpha_{3}+\alpha_{4}, \alpha=\alpha_{2}$.

3. The nine roots $\alpha_{2}, \alpha_{1}+\alpha_{2}, \alpha_{2}+\alpha_{3}, \alpha_{2}+\alpha_{4}, \alpha_{1}+\alpha_{2}+\alpha_{3}, \alpha_{1}+\alpha_{2}+\alpha_{4}, \alpha_{2}+\alpha_{3}+\alpha_{4}$, $\alpha_{1}+\alpha_{2}+\alpha_{3}+\alpha_{4}, \alpha_{1}+2 \alpha_{2}+\alpha_{3}+\alpha_{4}$ form the intersection $\lambda(w) \cap R^{4}$ for $R^{4} \subset R$ generated by $\left\{\alpha_{i}\right\}$.

The root $\alpha_{2}$ is always a simple root of $E_{6}$ but $\alpha_{1}, \alpha_{3}$ and $\alpha_{4}$ need not be. The three roots $\alpha_{1}, \alpha_{3}$ and $\alpha_{4}$ will not be elements of $\lambda(w)$. Together with the nine roots above these are 
all twelve positive roots in the $D_{4}$ subsystem $R^{4}$ determined by $\alpha_{1}, \alpha_{2}, \alpha_{3}, \alpha_{4}$, i.e., the condition nine but not twelve from (3.5) is satisfied.

Table 3 shows each of the Min-NGT words in $E_{6}$. Also included are the roots that make up the NGT and a choice of $\alpha_{1}, \alpha_{2}, \alpha_{3}, \alpha_{4}$ defining an appropriate $D_{4}$ subsystem. Since all of the words can not be written as compositions of pairwise orthogonal reflections, we uniformly put them in the shortlex form: the lexicographical ordering from left to right, but with the composition from right to left. We use a one-line representation of the roots from $E_{6}$ where the coefficient of the exceptional simple root is placed the last, i.e., $\begin{gathered}a b c d e \\ f\end{gathered}$ is written abcdef.

\section{Acknowledgements}

Partially supported by NSF grant DMS-0800642.

\section{References}

[1] Bourbaki N., Groupes et algèbres de Lie, Ch. 4-6, Hermann, Paris, 1969.

[2] Cherednik I., Non-semisimple Macdonald polynomials. I, Selecta Math., to appear, arXiv:0709.1742.

[3] Cherednik I., Factorizable particles on a half-line, and root systems, Teoret. Mat. Fiz. 61 (1984), 35-44 (in Russian).

[4] Cherednik I., Quantum Knizhnik-Zamolodchikov equations and affine root systems, Comm. Math. Phys. 150 (1992), 109-136.

[5] Cherednik I., Special bases of irreducible representations of a degenerate affine Hecke algebra, Funktsional. Anal. i Prilozhen. 20 (1986), no. 1, 87-88 (in Russian).

[6] Humphreys J.E., Reflection groups and Coxeter groups, Cambridge Studies in Advanced Mathematics, Vol. 29, Cambridge University Press, Cambridge, 1990.

[7] Kazhdan D., Lusztig G., Proof of the Deligne-Langlands conjecture for Hecke algebras, Invent. Math. 87 (1987), 153-215. 